\title{
CAPACIDADE FUNCIONAL E FORÇA MUSCULAR RESPIRATÓRIA COMO PREDITORES DE COMPLICAÇÕES EM PACIENTES SUBMETIDOS À REVASCULARIZAÇÃO MIOCÁRDICA
}

\author{
FUNCTIONAL CAPACITY AND RESPIRATORY \\ MUSCLE STRENGTH AS PREDICTORS OF \\ COMPLICATIONS IN PATIENTS SUBMITTED \\ TO MYOCARDIAL REVASCULARIZATION
}

Larissa Giovannetti Lunardi (Orcid: 0000-0003-4243-5750) ${ }^{1}$

Alana Kock Ferreguetti Costa (Orcid: 0000-0003-0888-2725)

\section{RESUMO}

Com o avanço dos métodos terapêuticos, a cirurgia de revascularização do miocárdio (CRVM) vem sendo realizada cada vez mais tardiamente. Desse modo, faz-se necessária uma avaliação prévia mais específica em busca dos pacientes mais vulneráveis a complicações pós-operatórias (PO), para avaliar se o desempenho no teste de caminhada de 6 minutos (TC6) e a pressão inspiratória máxima (PImáx) são capazes de predizer a ocorrência das principais complicações em pacientes submetidos à CRVM. Foi feita a análise de prontuários eletrônicos de um hospital referência em cardiologia, no período de julho a setembro de 2018. A análise estatística foi realizada utilizando os softwares Statistica 10 e Statistical Package of Social Sciences (SPSS) 20.0. Nível de significância p $<0,05$. Foram avaliados 25 prontuários, a maioria do sexo masculino, com idade média de 61,64 anos $\pm 8,82$. A distância média percorrida foi de $355,77 \mathrm{~m} \pm 87,461$ e a PImáx foi de $-62 \mathrm{cmH} 2 \mathrm{O} \pm 29,297$. Cada participante teve, em média, pelo menos duas complicações no PO $(1,92 \pm 1,187)$, sendo a hipocalemia $(\mathrm{p}=0,016)$ e o deficit perfusional $(\mathrm{p}=0,03)$ as que apresentaram correlação com a distância percorrida. Com relação à força muscular respiratória, a amostra se correlacionou apenas com as complicações pulmonares $(\mathrm{p}=0,002)$. O baixo desempenho no TC6 parece estar relacionado com o desenvolvimento de hipocalemia e deficit perfusional. Pacientes com força muscular $>-48 \mathrm{cmH} 2 \mathrm{O}$ apresentam maior risco de desenvolvimento de complicações pulmonares. Ademais, quanto menor a PImáx, maior o número de complicações pulmonares.

Palavras-chave: Revascularização Miocárdica; Ponte de Artéria Coronária; Teste de Caminhada; Pressões Respiratórias Máximas

\footnotetext{
ABSTRACT

With the advancement of therapeutic methods, myocardial revascularization surgery (CABG) has been performed more and more late. Thus, a more specific prior assessment is necessary in search of patients most vulnerable to postoperative complications (PO), to assess whether the performance in the 6-minute walk test (6MWT) and the maximum inspiratory pressure (MIP) are able to predict the occurrence of major complications in patients undergoing CABG. The analysis of electronic medical records of a reference hospital in cardiology was carried out from July to September 2018. The statistical analysis was performed using the software Statistica 10 and Statistical Package of Social Sciences (SPSS) 20.0. Significance level $\mathrm{p}<0.05$. Twenty-five medical records were evaluated, most of them male, with a mean age of 61.64 years \pm 8.82 . The average distance covered was $355.77 \mathrm{~m} \pm 87.461$ and the MIP was $-62 \mathrm{cmH} 2 \mathrm{O} \pm 29.297$. Each participant had, on average, at least two complications in the PO $(1.92 \pm 1.177)$, with hypokalemia $(\mathrm{p}=0.016)$ and perfusion deficit $(\mathrm{p}=0.03)$ being those that correlated with the distance covered. Regarding respiratory muscle strength, the sample correlated only with pulmonary complications $(\mathrm{p}=0.002)$. The low performance in the $6 \mathrm{MWT}$ seems to be related to the development of hypokalemia and perfusion deficit. Patients with muscle strength $>-48 \mathrm{cmH} 2 \mathrm{O}$ are at increased risk of developing pulmonary complications. In addition, the lower the MIP, the greater the number of pulmonary complications.

Keywords: Myocardial Revascularization; Coronary Artery Bypass; Walk Test; Maximal Respiratory Pressures.
} 


\section{INTRODUÇÃO}

A Doença Arterial Coronariana (DAC) pode ser definida como o deficit de irrigação sanguínea do coração por meio das artérias coronárias, estando diretamente relacionada com o grau de obstrução do fluxo sanguíneo. A principal consequência dessa obstrução é a redução do aporte de oxigênio ao coração, podendo levar a um quadro de angina ou Infarto Agudo do Miocárdio (IAM) ${ }^{1}$.

Após o IAM, a capacidade funcional dos pacientes pode ser limitada, sendo esta apontada como um fator prognóstico relevante em pacientes com doenças cardíacas ${ }^{2,3}$. Pode ser avaliada mediante aplicação de testes de esforço, como o teste de caminhada de 6 minutos (TC6), já recomendado pela Diretriz Europeia de Cirurgia de Revascularização Miocárdica $^{4}$ como uma alternativa eficaz e de baixo risco em pacientes cardiopatas. $\mathrm{O}$ TC6 tem sido amplamente utilizado para avaliar a morbimortalidade. Bittner et al. ${ }^{5}$ demonstraram que a distância percorrida em metros é inversamente proporcional à morbimortalidade por doenças cardíacas, ou seja, quanto maior a distância percorrida no teste, menor o índice de morbimortalidade.

A redução da capacidade pulmonar induzida pela cirurgia é outro fator que influencia na morbimortalidade e, consequentemente, no aumento dos custos hospitalares, bem como no número de readmissão hospitalar e no tempo prolongado de internação dessa população ${ }^{6}$.
O comprometimento da função pulmonar após a Cirurgia de Revascularização do Miocárdio (CRVM) tem caráter multifatorial, como, por exemplo, a utilização de anestesia geral, esternotomia mediana, manipulação torácica, uso da ventilação mecânica invasiva, utilização da circulação extracorpórea e, também, da condição pré-operatória ${ }^{7}$.

O mais indicado para reduzir as complicações pulmonares e funcionais seria o aperfeiçoamento da avaliação pré -operatória, para que medidas preventivas adequadas possam ser planejadas e, assim, direcionar a intervenção fisioterapêutica e a tomada de decisões clínicas ${ }^{6,8-10}$.

Considerando a influência do estado geral pré-operatório sobre o surgimento de complicações pós-operatórias $(\mathrm{PO})^{11}$, observou-se a necessidade de investigar se o TC6 e a manovacuometria, no pré-operatório, isoladamente, seriam capazes de detectar a ocorrência de complicações no PO. Diante do exposto, o presente trabalho tem como objetivo avaliar se o desempenho no TC6 e na manovacuometria é capaz de predizer a ocorrência das principais complicações em pacientes submetidos à CRVM com circulação extracorpórea. 


\section{MÉTODOS}

Trata-se de um estudo observacional retrospectivo com dados obtidos entre julho e setembro de 2018, por meio da análise de prontuário eletrônico $M V$ PEP de um hospital referência em cardiologia, em Vila Velha, Espírito Santo. Esta pesquisa foi aprovada pelo Comitê de Ética em Pesquisa da Faculdade Multivix, conforme resolução 466/12, nº $2.919 .506 / 2018$.

Foram incluídos no estudo indivíduos com faixa etária entre 18 anos e 85 anos, internados nas enfermarias ou unidades de terapia intensiva do Hospital Evangélico de Vila Velha, com diagnóstico de DAC e indicação de CRVM que tivessem descrito em prontuário os dados do TC6 e da manovacuometria. Foram excluídos do estudo pacientes retentores crônicos de gás carbônico $\left(\mathrm{CO}_{2}\right)$ ou que tivessem o diagnóstico de Doença Pulmonar Obstrutiva Crônica (DPOC); pacientes com histórico de cirurgia torácica prévia; uso de inotrópico no dia da realização do TC6 e o não cumprimento do protocolo institucional de ventilação não invasiva após extubação.

Foram classificados como critérios diagnósticos de complicações pulmonares no $\mathrm{PO}$ os indivíduos que evoluíram com infecção pulmonar, atelectasia, congestão pulmonar, edema pulmonar e/ou pneumotórax descritos em prontuário.

A inclusão da alteração perfusional capilar e da hipocalemia como complicações do PO foram baseadas nos critérios diagnósticos usados pela instituição. Para a disfunção perfusional capilar, considerou-se o tempo de enchimento capilar (TEC) maior que 4 segundos após a digitopressão do $2^{\circ}$ quirodáctilo sustentada por 20 segundos; alteração da tem- peratura das periferias e/ou cianose de extremidades em vigência de estratégias de aquecimento dos membros; e para a hipocalemia, nível de potássio abaixo de 135 $\mathrm{mEq} / \mathrm{L}$. Os critérios para incluir a retenção hídrica e complicações neurológicas foram, respectivamente, a partir da análise do prontuário: a descrição de edema intersticial no PO e a presença de deficit motor ou cognitivo na ausência dessas alterações relatadas previamente.

\section{Análise estatística}

A análise estatística descritiva foi realizada de acordo com a natureza das variáveis, apontando as contagens, porcentagens, média e desvio-padrão. Para comparação dos dados quantitativos, utilizou-se o Teste t; e para correlação entre essas variáveis, optou-se pela análise de correlação de Pearson. Para verificar a associação entre as variáveis categóricas, utilizou-se o teste de Qui-Quadrado de Pearson. Considerou-se nível de significância $p<0,05$. As análises foram realizadas utilizando os softwares Statistica 10 e Statistical Package of Social Sciences (SPSS) 20.0

\section{RESULTADOS}

Foram realizadas 53 CRVM no período de julho a setembro de 2018 , porém, de acordo com os critérios de exclusão do estudo, foram analisados 25 prontuários. A maioria dos pacientes inclusos no estudo era do sexo masculino (72\%). A distância média percorrida pelos indivíduos analisados corresponde aos níveis de caminhada 2 e 3 de acordo com a classificação de Bittner et al. ${ }^{5}$. Cada participante teve, em média, pelo menos duas complicações no PO como mostra a tabela 1 . 


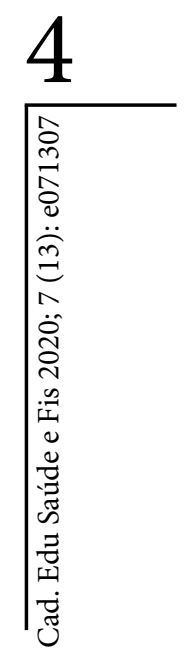

Tabela 1. Caracterização dos participantes da pesquisa.

\begin{tabular}{|c|c|c|}
\hline Variáveis & \multicolumn{2}{|c|}{ Média \pm DP } \\
\hline Idade (anos) & \multicolumn{2}{|c|}{$61,64 \pm 8,817$} \\
\hline $\operatorname{IMC}\left(\mathrm{kg} / \mathrm{m}^{2}\right)$ & \multicolumn{2}{|c|}{$27,38 \pm 4,477$} \\
\hline FEVE & \multicolumn{2}{|c|}{$56 \% \pm 13,564$} \\
\hline Tempo de CEC (min) & \multicolumn{2}{|c|}{$63,60 \pm 14,764$} \\
\hline No complicações & \multicolumn{2}{|c|}{$1,92 \pm 1,187$} \\
\hline PImáx $\left(\mathrm{cmH}_{2} \mathrm{O}\right)$ & \multicolumn{2}{|c|}{$-62,00 \pm 29,297$} \\
\hline Variáveis & $\mathbf{n}$ & $\%$ \\
\hline HAS & 18 & $72 \%$ \\
\hline DLP & 12 & $48 \%$ \\
\hline $\mathrm{DM}$ & 11 & $44 \%$ \\
\hline Tabagista & 12 & $48 \%$ \\
\hline Histórico familiar & 20 & $80 \%$ \\
\hline Evento cardiovascular prévio & 12 & $48 \%$ \\
\hline Sedentarismo & 21 & $84 \%$ \\
\hline \multicolumn{3}{|l|}{ Nível de caminhada } \\
\hline 1 & 6 & $24 \%$ \\
\hline 2 & 8 & $32 \%$ \\
\hline 3 & 9 & $26 \%$ \\
\hline 4 & 2 & $8 \%$ \\
\hline \multicolumn{3}{|l|}{ Classe Funcional } \\
\hline I & 4 & $16 \%$ \\
\hline II & 9 & $36 \%$ \\
\hline III & 10 & $40 \%$ \\
\hline IV & 2 & $8 \%$ \\
\hline
\end{tabular}

Valores expressos como média \pm desvio-padrão da média e porcentagem; Características clínicas da amostra avaliada exposta em média ou porcentagem; Idade: idade em anos; IMC: índice de massa corpórea; Classe funcional: Classe funcional segundo a New York Heart Association; HAS: Hipertensão arterial sistêmica; DLP: Dislipidemia; DM: Diabetes mellitus; Histórico familiar: Histórico familiar de doença cardiovascular; FEVE: Fração de ejeção de ventrículo esquerdo; Nível de caminhada: ponto de corte intuitivo para distância percorrida durante a caminhada de 6 minutos de acordo com Bittner et al, 1993 - Nível 1<300m, nível 2: 300m - 375m, nível 3: 375m - 450m, nível 4 > 450m; Tempo de CEC: Tempo de circulação extracorpórea em minutos; No complicações: número de complicações no período pós-operatório; PImáx: Pressão inspiratória máxima obtida através da manovacuometria. 
Tabela 2. Comparação entre a média do nível de caminhada e as complicações mais frequentes

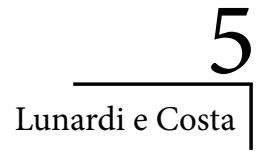

\begin{tabular}{lcc}
\hline \multicolumn{1}{c}{ Complicações frequentes } & Nível de caminhada & Média \pm DP \\
\hline Edema & $1,875 \pm 0,834$ & 0,141 \\
Hipocalemia & $1,500 \pm 0,548$ & $0,016^{*}$ \\
Perfusão capilar & $1,928 \pm 0,916$ & $0,03^{*}$ \\
Pulmonar & $2,060 \pm 0,961$ & 0,16 \\
\hline
\end{tabular}

DP: Desvio Padrão; ${ }^{\star}$ Estatisticamente significativo no nível de 5\%. Teste t para amostras independentes.

Tabela 3. Comparação entre Pressão Inspiratória Máxima e as complicações mais frequentes

\begin{tabular}{lcc}
\hline \multicolumn{1}{c}{ Complicações frequentes } & PImáx & $\boldsymbol{p}$ \\
\hline Edema & Média \pm DP & 0,64 \\
Hipocalemia & $-46,3 \pm 24,46$ & 0,733 \\
Neurológica & $-58,3 \pm 26,39$ & 0,506 \\
Perfusão capilar & $-54,0 \pm 27,01$ & 0,575 \\
Pulmonar & $-65,0 \pm 33,68$ & $0,002^{*}$ \\
\hline
\end{tabular}

DP: Desvio Padrão; ${ }^{\star}$ Estatisticamente significativo no nível de $5 \%$. Teste t para amostras independentes.

Figura 1. Correlação entre complicação pulmonar e Pressão Inspiratória Máxima (PImax)

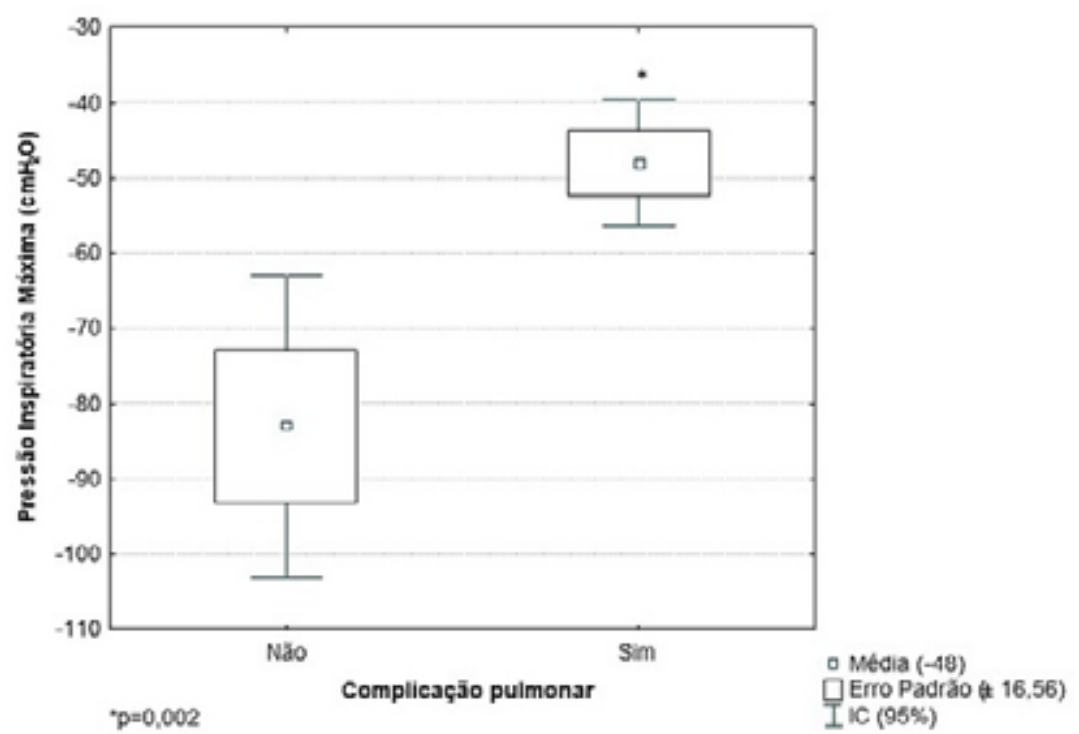


Figura 2. Correlação entre Pressão Inspiratória Máxima (PImax) e número de complicações

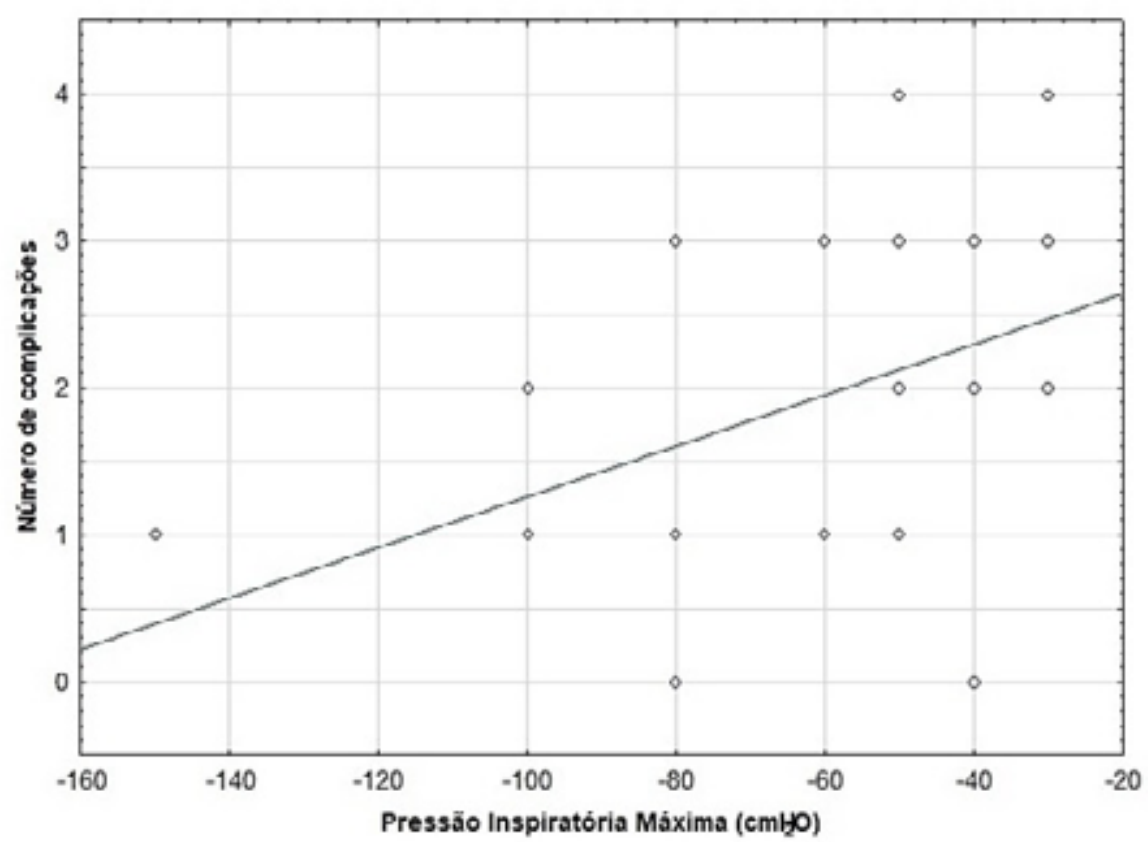

De acordo com o nível de caminhada, a maioria dos pacientes que apresentaram complicações no PO foram aqueles que caminharam até $375 \mathrm{~m}$ (nível de caminhada 1 e 2); e apenas a hipocalemia e o deficit perfusional periférico apresentaram valores significativos, ou seja, quanto menor a distância percorrida, maior a chance de apresentar essas complicações (tabela 2).

Em relação à força da musculatura respiratória, a Pressão Inspiratória Máxima (PImáx) apresentou correlação significativa apenas com as complicações pulmonares (tabela 3), em que os indivíduos que apresentam, em média, uma PImáx maior que - $48 \mathrm{cmH}_{2} \mathrm{O}$ tiveram complicações pulmonares no PO (figura 1).

Ainda, a PImax também se mostrou sensível ao número de complicações no PO, ou seja, quanto menor a força muscular, maior o número de complicaDISCUSSÃO

A partir do presente estudo, foram encontrados como principais resultados maioria do sexo masculino, com idade média de 61,64 anos $\pm 8,82$. A distância média percorrida foi de $355,77 \mathrm{~m}$ $\pm 87,461$ e a PImáx foi de $-62 \mathrm{cmH}_{2} \mathrm{O} \pm$ 29,297. Cada participante teve, em média, pelo menos duas complicações no PO $(1,92 \pm 1,187)$, sendo a hipocalemia $(\mathrm{p}=0,016)$ e o deficit perfusional $(\mathrm{p}=0,03)$ as que apresentaram correlação com a distância percorrida. Com relação à força muscular respiratória, a amostra se correlacionou apenas com as complicações pulmonares $(\mathrm{p}=0,002)$. $\mathrm{O}$ baixo desempenho no TC6 parece estar relacionado com o desenvolvimento de hipocalemia e deficit perfusional. Pacientes com força muscular $>-48 \mathrm{cmH}_{2} \mathrm{O}$ apresentam maior risco de desenvolvimento de complicações pulmonares. 
Observou-se que uma das causas da redução da capacidade ao exercício observada no teste de caminhada pode ser explicada por uma inadequada perfusão sanguínea, que é controlada de acordo com as demandas metabólicas e oxidativas do tecido. Essa disfunção está associada à fadiga muscular, um dos sintomas mais prevalentes e debilitantes associados à baixa qualidade de vida e à redução da atividade física em pacientes com $\mathrm{DAC}^{12-}$ 14 .

Pacientes portadores de cardiopatia isquêmica apresentam uma alteração da regulação entre a oferta e a demanda de oxigênio, levando, entre outras complicações, à angina e, consequentemente, ao desuso da musculatura periférica, prejudicando sua perfusão e a sua capacidade ao exercício ${ }^{15-17}$. Portanto, acredita-se que o elevado estresse físico e inflamatório causado pela cirurgia cardíaca seja capaz de prejudicar ainda mais a perfusão capilar ${ }^{13}$.

No pós-operatório, a fim de aumentar a concentração de oxigênio tecidual, uma das medidas adotadas é aumentar a sua oferta. Porém, esse quando dissolvido não é capaz de aumentar sua saturação, tendo como efeitos colaterais a vasoconstrição e o aumento do estresse oxidativo, podendo agravar a resposta inflamatória sistêmica e, consequentemente, aumentar a morbimortalidade ${ }^{17,18}$.

Riedi et al. ${ }^{19}$ afirmam que o estresse ao qual o paciente é submetido durante a cirurgia é capaz de reduzir o nível sérico de potássio. Sabe-se ainda que pacientes portadores de cardiopatia isquêmica, por apresentam aumento da demanda energética, podem apresentar redução da capacidade funcional por reduzir o transporte ativo da bomba de sódio e potássio, alterando a concentração de potássio no sangue $^{20}$. Pequenas distâncias percorridas no TC6 $(<325 \mathrm{~m})$ parecem indicar uma maior susceptibilidade a distúrbios eletrolíticos, pois o potássio possui um papel fundamental para o funcionamento das células, bem como para manutenção do potencial elétrico transmembrana, possibilitando a transmissão nervosa adequada, contração muscular e tônus vascular ${ }^{20}$. Já é conhecido na literatura que pacientes com alterações bioquímicas e eletrolíticas apresentam uma capacidade física reduzida e, consequentemente, alteração da contração muscular e capacidade de realização do exercício ${ }^{21}$.

Em relação às complicações pulmonares, o presente estudo encontrou resultados estatisticamente relevantes quando comparado a PImáx, o que diverge dos dados encontrados na literatura. Riedi et al. ${ }^{19}$ analisaram a força muscular respiratória de 63 indivíduos submetidos à cirurgia cardíaca. Como resultado, não encontraram relação entre a força muscular respiratória no pré-operatório e as complicações PO. Outro estudo avaliou a força muscular respiratória e sua influência na incidência de complicações pulmonares em pacientes submetidos à cirurgia cardíaca. Como desfecho, os autores afirmam que a força muscular não pode ser considerada um fator de risco para o desenvolvimento de complicações pulmonares no $\mathrm{PO}^{22}$. 
Schnaider et al. ${ }^{23}$ encontraram em seu estudo uma tendência que poderia indicar uma associação entre a força muscular e as complicações pulmonares no PO, porém, sem valores estatisticamente relevantes. Apesar de os estudos não apontarem uma correlação, vários fatores têm sido sugeridos como causas para influenciar o desenvolvimento de tais complicações, incluindo o tipo e a duração da cirurgia, a esternotomia, a dor, o dreno, bem como a efeito anestésico e a condição pré-operatória do paciente $e^{7,25,26}$.

Estudos têm apontado que os músculos respiratórios desempenham um papel vital na manutenção da estrutura e na permeabilidade das vias aéreas superiores, pois são responsáveis por realizar a manutenção das pressões das vias aéreas. Em caso de disfunções e/ou obstruções das vias aéreas, aumenta-se o risco de complicações pulmonares ${ }^{27}$.

Este estudo comprova a correlação entre fraqueza respiratória e presença de complicações pulmonares, mostrando a importância de um trabalho prévio à cirurgia com fortalecimento pulmonar, preparando melhor esse paciente com intuito de reduzir complicações e, consequentemente, tempo de internação e custos hospitalares ${ }^{3}$. Não apenas a fraqueza muscular respiratória tem sido abordada no paciente cardiopata. ${ }^{28}$ Novos estudos têm identificado o desenvolvimento da síndrome da fragilidade em pacientes cada vez mais jovens, que consiste em desnutrição crônica, sarcopenia, fraqueza muscular e intolerância ao exercício ${ }^{29,30}$. Essa síndrome também pode contribuir para o desenvolvimento de complicações no período pós-operatório de cirurgias cardíacas $^{8,11}$.
É importante mencionar que alguns fatores limitaram a aquisição de dados para este estudo: o curto período disponível para a coleta de dados e a falta ou inadequação no registro de informações no prontuário eletrônico. Dada a importância do tema e a divergência de resultados, torna-se essencial realizar novas pesquisas para investigar a influência da força muscular respiratória e da capacidade funcional como fatores de risco para o desenvolvimento de complicações no PO de CRVM.

\section{CONCLUSÃO}

O baixo desempenho de pacientes coronariopatas no TC6 durante o pré-operatório de revascularização do miocárdio parece estar relacionado com o desenvolvimento de hipocalemia e deficit perfusional, sendo as complicações mais comuns evidenciadas no PO. Pacientes com força muscular maior que $-48 \mathrm{cmH} 2 \mathrm{O}$ apresentam maior risco de desenvolvimento de complicações pulmonares. Para este estudo, quanto menor a força muscular respiratória, maior o número de complicações pulmonares no PO. 


\section{REFERÊNCIAS}

1. Pinho RA, De Araújo MC, Ghisi GLDM, Benetti M. Doença Arterial Coronariana, Exercício Físico e Estresse Oxidativo. Arq Bras Cardiol 2010; 94(4):549-55. doi: 10.1590/S0066782X2010000400018

2. Diniz LS. Segurança da realização precoce do teste de caminhada de seis minutos após infarto agudo do miocárdio [dissertação] [internet]. Belo Horizonte (MG): Universidade Federal de Minas Gerais; 2014. Disponível em: http://www.bibliotecadigital.ufmg.br/ dspace/bitstream/handle/1843/BUBD9MRH94/disserta_o_de_mestrado 1 via santos diniz 2014.pd$\underline{\mathrm{f}}$ sequence $=1$

3. Castro I, Batlouni M, Cantarelli E, Ramires JAF, Luna RL, Feitosa GS. Cardiologia: Princípios e prática. $1^{\mathrm{a}}$ ed. Porto Alegre. ArtMed; 1999. v.1. 1277 p.

4. Wijns W, Kolh P, Danchin N, Di Mario C, Falk V, Folliguet T, et al. Guidelines on myocardial revascularization. The Task Force on Myocardial Revascularization of the European Society of Cardiology (ESC) and the European Association for Cardio-Thoracic Surgery (EACTS). Eur Heart J 2010; 31:250155. doi: 10.1093/eurheartj/ehy394

5. Bittner V, Weiner DH, Yusuf S, Rogers WJ, Mcintyre KM, Bangdiwala SI, et al. Prediction of Mortality and Morbidity With a 6-Minute Walk Test in Patients With Left Ventricular Dysfunction. JAMA Cardiol 1993; 270(14):1702-7. doi: 10.1001/ jama.1993.03510140062030

6. Sabaté S, Mazo V, Canet J. Predicting postoperative pulmonary complications: implications for outcomes and costs. Curr Opin Anesth 2014; 27:201-9. doi: 10.1097/ACO.0000000000000045.
7. Oliveira EK, Silva VZM, Turquetto ALR. Relação do teste de caminhada pós-operatório e pulmonary function com o tempo de internação da cirurgia cardíaca. Rev Bras Cir Cardiovasc 2009; 24(4):478-84. doi: 10.1590/S010276382009000500008

8. Bottura C. Avaliação da fragilidade em indivíduos submetidos à cirurgia cardíaca [tese]. Ribeirão Preto (SP): Faculdade de Medicina de Riberirão Preto; 2017. doi: 10.11606/D.17.2018. tde-06042018-095710

9. Hulzebos EHJ, Meeteren NLU Van, Bie RA De, Dagnelie PC, Helders PJM. Prediction of Postoperative Pulmonary Complications on the Basis of Preoperative Risck Factors in Patients Who Had Undergone Coronary Artery Bypass Graft Surgery. Phys Ther 2003; 83(1):8-16. doi: 10.1093/ptj/83.1.8

10. Kovacs J, Moraru L, Antal K, Cioc A, Voidazan S, Szabo A. Are frailty scales better than anesthesia or surgical scales to determine risk in cardiac surgery. Korean J Anesthesiol 2017; 70(2):15762. doi: 10.4097/kjae.2017.70.2.157.

11. Gualandro DM, Yu PC, Caramelli B, Marques AC, Calderaro D, Fornari LS. $3^{\text {a }}$ Diretriz de Avaliação Cardiovascular Perioperatória da Sociedade Brasileira de Cardiologia. Arq Bras Cardiol 2017 set; 109(3,Supl I):2-8. doi: 10.5935/ abc. 20170140

12. Eckhardt AL, Devon HA, Piano MR, Ryan CJ, Zerwic JJ. Fatigue in the presence of coronary heart disease. Nurs Res 2014; 63(2):83-93. doi: 10.1097/ NNR.0000000000000019

13. Borges JA, Quintão MMP, Chermont SSMC, Mendonça Filho HTF, Mesquita ET. Fadiga: Um Sintoma Complexo e seu Impacto no Câncer e na Insuficiência Cardíaca. Int J Cardiovasc Sci 2018; 31(4):433-42. doi: 10.5935/23594802.20180027 
14. Ribeiro JP, Clausell N. Em busca de novos paradigmas para o manejo da cardiopatia isquêmica. Arq Bras Cardiol 1997; 69(1):1-12. doi: 10.1590/ S0066-782X1997000700001

15. Moreu-Burgos J, Macaya-Miguel C. Fisiopatología del miocardio isquémico. Importancia de la frecuencia cardiaca. Rev Española Cardiol Supl 2007; 7:19D-25D. doi: 10.1016/S11313587(07)75772-2

16. Spoelstra-De Man AME, Smit B, Oudemans-Van Straaten HM, Smulders YM. Cardiovascular effects of hyperoxia during and after cardiac surgery. Anaesthesia 2015; 70(11):1307-19. doi: 10.1111/anae. 13218

17. De Blasi RA, Romagnoli S, Rocco M. Bedside Assessment of the Microvascular Venous Compartment in Cardiac Surgery Patients With Valvular Diseases Undergoing Cardiopulmonary Bypass. J Cardiothorac Vasc Anesth. Elsevier Inc.; 2017; 31(1):105-14. doi: 10.1053/j.jvca.2016.06.001

18. Cuppari L, Bazanelli AP. Funções plenamente reconhecidas de nutrientes: potássio. São Paulo. Força-tarefa - Alimentos fortificados e suplementos. [internet]. Comitê de Nutrição ILSI Brasil, 2010. Disponível em: http:// ilsi.org/brasil/wp-content/uploads/ sites/9/2016/05/11-Pota\%CC\%81ssio. pdf

19. Riedi C, Mora CTR, Driessen T, Coutinho $\mathrm{M}$ de CG, Mayer DM, Moro FL, et al. Relação do comportamento da força muscular com as complicações respiratórias na cirurgia cardíaca. Rev Bras Cir Cardiovasc 2010; 25(4):500-5. doi: 10.1590/S010276382010000400013 .
20. Dipp T, Silva AMV, Signori LU, Strimban TM, Nicolodi G, Graciele S, et al. Força Muscular Respiratória e Capacidade Funcional na Insuficiência Renal Terminal. Rev Bras Med Esporte 2010; 16(4):246-9. doi: 10.1590/S151786922010000400002

21. Su H. Hypokalemia in cardiac surgery under cardiopulmonary bypass (CPB). In: International Practice in Cardiothoracic Surgery. 2011th ed. Springer Netherlands; 1986. p. 145-160. doi: 10.1007/978-94-009-4259-2 16

22. Bastos TAB, Melo VA, Silveira FS, Guerra DR. Influência da força muscular respiratória na evolução de pacientes com insuficiência cardíaca após cirurgia cardíaca. Rev Bras Cir Cardiovasc 2011; 26(3):355-63. doi: 10.5935/16789741.20110009

23. Schnaider J, Karsten M, Carvalho T, Lima WC. Influência da força muscular respiratória pre operative na evolução clínica após coronary artery bypass surgery. Fisioter e Pesqui 2010; 17(1):52-7. doi: 10.1590/S1809 29502010000100010

24. Rouhi-Boroujeni H, Rouhi-Boroujeni H, Rouhi-Boroujeni P, Sedehi M. Long-term pulmonary functional status following coronary artery bypass grafting surgery. ARYA Atheroscler 2015;11(2):163-6. Disponível em: https://www.ncbi.nlm.nih.gov/pmc/articles/PMC4568203/

25. Westerdahl E, Jonsson M, Emtner M. Pulmonary function and health-related quality of life 1-year follow up after cardiac surgery. J Cardiothorac Surg 2016; 11(99):1-8. doi: 10.1186/s13019-0160491-2 
26. Sasaki N, Meyer MJ, Eikermann M. Postoperative Respiratory Muscle Dysfunction. Anesthesiology 2013;118(4):961-78. doi: 10.1097/ ALN.0b013e318288834f

27. Gomes Neto M, Martinez BP, Reis HFC, Carvalho VO. Pre- and postoperative inspiratory muscle training in patients undergoing cardiac surgery: Systematic review and meta-analysis. Clin Rehabil 2016; 31(4):454-64. doi: $10.1177 / 0269215516648754$

28. Afilalo J, Kim S, O’Brien S, Brennan JM, Edwards FH, Mack MJ, et al. Gait speed and operative mortality in older adults following cardiac surgery. JAMA Cardiol 2016; 1(3):314-21. doi: 10.1001/jamacardio.2016.0316

29. Ferriolli E, Pessanha FPA dos S, Moreira VG, Dias RC, Neri AL, Lourenço RA. Body composition and frailty profiles in Brazilian older people: Frailty in Brazilian Older People Study-FIBRA-BR. Arch Gerontol Geriatr 2017; 71:99104. doi: 10.1016/j.archger.2017.03.008

30. Hamilton W, Round J. Identifying frailty in primary care. BMJ 2017; 358(j4478):1-2. doi: 10.1136/bmj.j4478

Recebido: 13/07/2019

Aprovado: 20/05/2020 